\title{
LINEAR SYMMETRIES OF FREE BOSON FIELDS $\left(^{1}\right)$
}

\author{
BY \\ DAVID SHALE
}

1. Introduction. 1.1. The real normalizable solutions of the Klein-Gordan equation: $\square \psi=m^{2} \psi, m>0$; form a real Hilbert space $K . K$ admits a nonsingular skew 2 -form $B(\cdot, \cdot)$ which is uniquely determined, apart from a scalar factor, by the condition that it be invariant under the canonical action of the proper inhomogeneous Lorentz group on $K$. There is an orthogonal transformation $\Lambda$ on $K$, commuting with this action, which when interpreted as multiplication by $i$ allows $K$ to be made into a complex Hilbert space $H$ with $B(\cdot, \cdot)$ as the imaginary part of the inner product. To quantize the KleinGordan Field, one needs only $K$ and $B(\cdot, \cdot)$, or equivalently, $H$. Similarly a complex Hilbert space determines the quantization of every free boson field $[11 ; 12]$.

Accordingly, following Segal [11], we give:

Definition 1.1. For any complex Hilbert space $H, \Sigma(H)$ the Single Particle Structure (or Classical Field), determined by $H$, is the pair $\{K, B(\cdot, \cdot)\}$; where $K$ is $H$ considered as a real Hilbert space with inner product $\left(z_{1}, z_{2}\right)$ $=\operatorname{Real}\left(z_{1}, z_{2}\right)_{c}$, where $\left(z_{1}, z_{2}\right)_{c}$ is the inner product in $H$, and $B(\cdot, \cdot)$ is the nonsingular skew 2 -form defined by $B\left(z_{1}, z_{2}\right)=\operatorname{Im}\left(z_{1}, z_{2}\right)_{c}$.

Definition 1.2. A quantization of $\Sigma(H)$ is a (strongly) linear map $R(\cdot)$ from $K$ to selfadjoint operators on a complex Hilbert space such that if $V(z)$ $=\exp (i R(z))$, then $V(\cdot)$ is weakly continuous when restricted to any finite dimensional subspace of $K$ and the following commutation relations are satisfied:

$$
V\left(z_{1}\right) \cdot V\left(z_{2}\right)=e^{-i B\left(z_{1}, z_{2}\right) / 2} \cdot V\left(z_{1}+z_{2}\right), \quad \text { for all } z_{1}, z_{2} \in K .
$$

The above commutation relations are essentially those given by Weyl [17]. In the present form they have been used by von Neumann [16], for finite systems and by Segal [11], for fields. Quantization as defined above is formally implied by the current physical formalism which assigns to each point in space-time an "operator-valued-distribution" and has the advantage of being rigorous.

The symmetries that concern us are those that arise as linear transformations of the wave functions of the classical field. Specifically we consider the multiplicative group of all bounded linear transformations $T$ on $K$ such that if $R(\cdot)$ is a quantization then $z \rightarrow R(T z)$ is also. (See [11].) This is the group defined equivalently by:

Received by the editors May 31, 1961.

(1) The contents of this paper is approximately the same as that of the author's doctoral dissertation at the University of Chicago. 
Definition 1.3. The Symplectic group $S p(K)$ determined by the single particle structure $\Sigma(H)$, is the multiplicative group of real bounded linear transformations on $K$ which preserve $B(\cdot, \cdot)$.

When the dimension of $H$ is not finite there are at least continuumly many irreducible unitarily inequivalent quantizations of $\Sigma(H)$ [10]. Consequently for a given irreducible quantization $R(\cdot)$ it is of interest to determine the subgroup of $S p(K)$ consisting of those $T$ for which there is a unitary transformation $Y(T)$ such that $R(T z)=Y(T) \cdot R(z) \cdot Y(T)^{-1}$ for all $z \in K$. Using the theory of integration over Hilbert space [especially 10, Theorem 3 ] we show (Theorem 4.1 below) that in the case of the Fock-Cook quantization [3] the subgroup is $\left\{T:\left(T^{*} \cdot T\right)^{1 / 2}-I\right.$ is Hilbert-Schmidt $\}$. We study the resulting projective unitary representation.

The choice of the Fock-Cook quantization is not arbitrary. It appears likely that for the free scalar meson and Maxwell fields the only pure regular state which is Lorentz invariant and such that in the quantization determined by it the energy is positive, is the zero-interaction vacuum state which determines the Fock-Cook quantization (cf. [13]).

Summary of contents. In $\$ 2$ we introduce the various classes of groups needed later together with some of their simple properties. $\$ 3$ is concerned with the action of the general linear group of operators on a real Hilbert space $M$ on the normal distribution over $M$. Both sections are necessary preliminaries to $\S 4$ where we treat the main problem. In $\S 5 H$ is restricted to be of finite dimension. By the well-known theorem of Stone and von Neumann any two irreducible quantizations are unitarily equivalent [16]. Consequently the resulting projective unitary representation of $S p(K)$ is essentially unique. We show that it determines a double-valued unitary representation of $S p(K)$. Now quantization here has as its fermion analogue the representation of the Clifford algebra over $K$ by spinors. The analogue of our representation is the spinor representation of the orthogonal group on $K$ due to Brauer and Weyl [2]. In infinitesimal form our result has been obtained by van Hove [15]. In $\$ 6$ we apply our techniques to prove some theorems about the transformation properties of the states and observables of free boson fields.

Acknowledgment. The author wishes to express his gratitude to Professor I. E. Segal for many helpful suggestions and conversations.

2. Preliminaries on groups of operators. 2.1. We introduce some infinite dimensional analogues of the General Linear Group. Let $X$ be a bounded linear operator on a Hilbert space $H$ which may be real or complex and of arbitrary dimension. $X$ is elementary if $X=X_{1} \oplus 0$ when $H$ is decomposed suitably as $H_{1} \oplus H_{1}^{\perp}$, with $\operatorname{dim} H_{1}$ finite. Let $\left\{e_{\alpha}\right\}$ be an arbitrary orthonormal basis for $H$. Then $X$ is Hilbert-Schmidt if its $L_{2}$ norm $\|X\|_{2}=\left(\sum_{\alpha}\left\|X e_{\alpha}\right\|^{2}\right)^{1 / 2}$ is finite. The Hilbert-Schmidt operators with this norm form a Banach space which will be denoted $L_{2}$. Similarly the operators of trace class: $\left\{X:\|X\|_{1}\right.$ 
$=\sup (U$ unitary $) \sum_{\alpha}\left|\left(U X e_{\alpha}, e_{\alpha}\right)\right|$ is finite $\}$ form a Banach algebra denoted by $L_{1}$. Both are independent of the choice of basis. Now let $G L(H)$ General Linear Group on $H$ be the multiplicative group of all bounded linear operators on $H$ with bounded inverses. When $\operatorname{dim} H$ is not finite we consider as well as $G L(H)$ itself, the subgroups given by:

Definition 2.1. For any Hilbert space $H$, the group of Tame Operators $G L(H)_{0}$, the $L_{1}$ Group $G L(H)_{1}$, and the $L_{2}$ Group $G L(H)_{2}$ are the subgroups of $G L(H)$ whose elements have the form $I+X$ where $X$ is elementary, of trace class and Hilbert-Schmidt respectively. For any operator $T$, let $u(T)$ and $|T|$ be the isometric and selfadjoint parts of the polar decomposition. The Restricted General Linear Group $r G L(H)$ is all operators in $G L(H)$ such that $|T| \in G L(H)_{2}$.

Let $G L(H)_{1}$ and $G L(H)_{2}$ have the topologies arising from $L_{1}$ and $L_{2}$ respectively. $G L(H)_{0}, G L(H)_{1}$ and $G L(H)_{2}$ are normal subgroups of $G L(H)$ and each is dense in the next. Applications will be mainly with $r G L(H)$. We include $G L(H)_{2}$ as a natural subgroup of $r G L(H) . G L(H)_{0}$ is the most conservative extension from the finite dimensional classical group. The determinant in the finite case extends at once to a multiplicative function $\Delta(\cdot)$ on $G L(H)_{0}$. Our inclusion of $G L(H)_{1}$ is explained by:

Lemma 2.1. (a) $\Delta(\cdot)$ extends uniquely to a continuous representation on $G L(H)_{1}$.

(b) There is no continuous local representation of $G L(H)_{2}$, or of its unitary subgroup $U(H)_{2}$, which coincides with $\Delta(\cdot)$ on the tame operators.

Proof. (a) Since $G L(H)_{0}$ is dense in $G L(H)_{1}$ we need only show that $\Delta(\cdot)$ is continuous when $G L(H)_{0}$ is topologised as a subset of $G L(H)_{1}$. Since $G L(H)_{0}$ is a topological group in this topology it is enough to prove continuity at $I$. If $H$ has finite dimension, then on a suitable neighbourhood $N$ of $I, \Delta(T)$ $=\exp (\operatorname{tr}(\log (T)))$ where $\operatorname{tr}(\cdot)$ is the trace and $\log (\cdot)$ is defined by the usual power series. The same remark holds for $G L(H)_{0}$ in the general case. Continuity follows since $\log (\cdot)$ is continuous from $N$ to $L_{1}$ and $\operatorname{tr}(\cdot)$ is continuous on $L_{1}$.

(b) Let $N(\epsilon)=\left\{T \in G L(H)_{2}:\|T-I\|_{2}<\epsilon, \epsilon>0\right\}$ be an arbitrarily small neighbourhood of $I$. Let $\left\{e_{n}\right\}$ be an orthonormal sequence in $H$ and $\left\{a_{n}\right\}$ a sequence of numbers such that $\sum_{n} a_{n}^{2}<\infty$ and $\sum_{n} a_{n}$ diverges. Let $A_{m}$ be such that $A_{m} e_{n}=a_{n} e_{n}$ all $n<m$, and $A_{m} f=0$ for all $f \perp\left\{e_{m}: m<n\right\}$. It may be seen that $\exp \left(i A_{m}\right) \in U(H)_{2} \cap N(\boldsymbol{\epsilon}), m=1, \cdots, \infty$, that the $\exp \left(i A_{m}\right)$ are tame and converge to $\exp \left(i A_{\infty}\right)$ in $G L(H)_{2}$ and that $\left\{\Delta\left(\exp \left(i A_{m}\right)\right)\right\}$ diverges.

Our discussion of $r G L(H)$ will be for $H$ real. The two most important subgroups are the group of orthogonal operators $O(H)$ and $G L(H)_{2}$. Polar decomposition gives the unique decomposition $r G L(H)=O(H) \times G L(H)_{2}^{+}$, where ${ }^{+}$indicates the subset of positive selfadjoint operators. Under the weak operator topology which coincides here with the strong operator topology 
$O(H)$ is a topological group. It seems appropriate therefore to give $O(H)$ this topology, topologise $G L(H)_{2}^{+}$as a subset of $G L(H)_{2}$ and $r G L(H)$ as the cartesian product of these. Any continuous representation of $r G L(H)$ is then automatically continuous on $O(H)$ and $G L(H)_{2}$.

LEMMA 2.2. $r G L(H)$ is a topological group.

If for an operator $X$ on $H$, there is an orthonormal basis $\left\{e_{\alpha}\right\}$, and numbers $\left\{\lambda_{\alpha}\right\}$, such that $X e_{\alpha}=\lambda_{\alpha} e_{\alpha}$ for all $\alpha$, then $\left\{e_{\alpha}\right\}$ is an eigenbasis and $\left\{e_{\alpha}, \lambda_{\alpha}\right\}$ an eigensystem for $X$.

Sublemma. (a) For fixed $X \in L_{2}^{+}$, the map $U \rightarrow U^{-1} \cdot X \cdot U$ from $O(H)$ to $L_{2}^{+}$ is continuous at I.

(b) The map $(U, T) \rightarrow U^{-1} \cdot T \cdot U$ from $O(H) \times G L(H)_{2}^{+}$to $G L(H)_{2}^{+}$, is continuous.

Proof of sublemma. (a) Since $X$ is Hilbert-Schmidt it is completely continuous and hence possesses an eigensystem $\left\{e_{\alpha}, \lambda_{\alpha}\right\}$. There is no essential loss in supposing that $\alpha$ ranges over the positive integers. Given $\epsilon>0$, let $n$ be chosen so that $\sum_{n+1}^{\infty} \lambda_{\alpha}^{2}<\epsilon$. Let $0<\delta<1$. Let $V \in O(H)$ be such that $\left\|V e_{\alpha}-e_{\alpha}\right\|<\delta$ for $\alpha \leqq n$. Since $\|x-y\|^{2} \leqq 2\|x\|^{2}+2\|y\|^{2}$, it follows that for $\alpha \leqq n$,

$$
\begin{aligned}
\left\|X \cdot V e_{\alpha}-V \cdot X e_{\alpha}\right\|^{2} & \leqq 2\left\|X(V-I) e_{\alpha}\right\|^{2}+2\left\|(V-I) X e_{\alpha}\right\|^{2} \\
& \leqq 2\|X\|^{2} \cdot \delta^{2}+2 \lambda_{\alpha}^{2} \delta^{2} \leqq 4\|X\|_{2}^{2} \delta^{2}
\end{aligned}
$$

Also

$$
\begin{aligned}
\sum_{n+1}^{\infty}\left\|X \cdot V e_{\alpha}\right\|^{2} & =\|X\|_{2}^{2}-\sum_{1}^{n}\left\|X \cdot V e_{\alpha}\right\|^{2} \\
& \leqq \sum_{1}^{n}\left|\left\|X e_{\alpha}\right\|^{2}-\left\|X \cdot V e_{\alpha}\right\|^{2}\right|+\sum_{n+1}^{\infty}\left\|X e_{\alpha}\right\|^{2} .
\end{aligned}
$$

Now $\left|\left\|X e_{\alpha}\right\|^{2}-\left\|X \cdot V e_{\alpha}\right\|^{2}\right| \leqq\left\|X(V-I) e_{\alpha}\right\| \cdot\left(\left\|X e_{\alpha}\right\|+\left\|X \cdot V e_{\alpha}\right\|\right) \leqq 2\|X\|_{2}^{2} \delta$, for all $\alpha \leqq n$. It follows that the last term above is less than $2 n\|X\|_{2}^{2} \delta+\epsilon$. Hence

$$
\begin{aligned}
\left\|V^{-1} \cdot X \cdot V-X\right\|_{2}^{2} & =\|X \cdot V-V \cdot X\|_{2}^{2} \\
& =\sum_{1}^{n}\left\|X \cdot V e_{\alpha}-V \cdot X e_{\alpha}\right\|^{2}+\sum_{n+1}^{\infty}\left\|X \cdot V e_{\alpha}-V \cdot X e_{\alpha}\right\|^{2} \\
& \leqq 4 n\|X\|_{2}^{2} \delta^{2}+2 \sum_{n+1}^{\infty}\left\|X \cdot V e_{\alpha}\right\|^{2}+2 \sum_{n+1}^{\infty}\left\|V \cdot X e_{\alpha}\right\|^{2} \\
& \leqq 4 n\|X\|_{2}^{2} \cdot \delta^{2}+\left(2 n\|X\|_{2}^{2} \delta+\epsilon\right)+\epsilon=6 n\|X\|_{2}^{2} \delta+2 \epsilon .
\end{aligned}
$$


(b) For $(U, T)$ and $\left(U_{1}, T_{1}\right)$ in $O(H) \times G L(H)_{2}^{+}$we have,

$$
\begin{aligned}
& \left\|U_{1}^{-1} \cdot T_{1} \cdot U_{1}-U^{-1} \cdot T \cdot U\right\|_{2} \\
& \quad \leqq\left\|U_{1}^{-1} \cdot\left(T_{1}-T\right) U_{1}\right\|_{2}+\left\|U_{1}^{-1} \cdot T \cdot U_{1}-U^{-1} \cdot T \cdot U\right\|_{2} \\
& \quad=\left\|T_{1}-T\right\|_{2}+\|X \cdot V-V \cdot X\|_{2},
\end{aligned}
$$

where $X=T-I$ and $V=U_{1} \cdot U^{-1}$. The result now follows from part (a) and the fact that $O(H)$ is a topological group.

Proof of Lemma 2.2. Let $T, T_{1} \in r G L(H)$. We have $T^{-1}=u(T)^{-1}$ $\cdot\left(u(T) \cdot|T|^{-1} \cdot u(T)^{-1}\right)=u\left(T^{-1}\right) \cdot\left|T^{-1}\right|$. The continuity of $T \rightarrow T^{-1}$ now follows from part (b) of the sublemma and the continuity of the operations of taking inverses in $O(H)$ and $G L(H)_{2}$. It may be seen that the operations: $T \rightarrow T^{*}$ and $T \rightarrow T^{1 / 2}$ are continuous in $G L(H)_{2}$ and $G L(H)_{2}^{+}$respectively and hence that polar decomposition is continuous in $G L(H)_{2}$ and that $T \rightarrow u(T)$ is continuous from $G L(H)_{2}$ to $O(H)$. Hence observing that $u\left(T \cdot T_{1}\right)=u(T)$ $\cdot u\left(T_{1}\right) \cdot u\left(\left[u\left(T_{1}\right)^{-1} \cdot|T| \cdot u\left(T_{1}\right)\right] \cdot\left|T_{1}\right|\right)$ and $\left|T \cdot T_{1}\right|=\mid\left(u\left(T_{1}\right)^{-1} \cdot|T| \cdot u\left(T_{1}\right)\right)$ $\cdot\left|T_{1}\right| \mid$, and using the Sublemma we may establish continuity of multiplication in $r G L(H)$. Details are omitted.

2.2. Let $H$ be complex. We now consider the corresponding classes of symplectic groups over $\Sigma(H)$.

Definition 2.2. $S p(K)_{i}=S p(K) \cap G L(K)_{i}$ for $i=0,1,2$. The restricted symplectic group $r S P(K)$ is $S p(K) \cap r G L(K)$. Each Symplectic group is topologised as a subspace of the corresponding general linear group.

It may be seen that the group of operators which are orthogonal and symplectic is $U(H)$. Let $\Lambda$ be the orthogonal operator on $K$ defined by $\Lambda z=i z$. Then $\Lambda^{2}=-1$. A straightforward computation shows that a regular operator $T$ on $K$ is symplectic if and only if $\Lambda \cdot T \cdot \Lambda^{-1}=T^{*-1}$. It may be seen that if $T \in S p(K)^{+}$then the multiplicity of 1 in the point spectrum of $T$ is zero, even or infinite. It follows readily that $K$ may be decomposed as $K_{1} \oplus K_{2}$ with $\Lambda^{-1} K_{2}=K_{1}$ so that $T=\Lambda^{-1} \cdot S^{-1} \cdot \Lambda \oplus S$ with $S \in G L(K)_{2}^{+}$. Conversely any $T$ with this form is in $S p(K)_{2}^{+}$. It follows readily that $S p(K)$ is stable under polar decomposition.

Notation. For each single particular structure $\Sigma(H)$ let $M$ be a fixed subspace of $K$ such that $K=\Lambda^{-1} M \oplus M$. For convenience this will be written $K=M \oplus M$. Then for $z \in K, z=x \oplus y$ with $x, y \in M$ and $\Lambda z=-y \oplus x$.

Lemma 2.3. (a) If $T \in S p(K)^{+}\left(r e s p . T \in S p(K)_{2}^{+}\right)$then there is a $U \in U(H)$ such that $T=U^{-1} \cdot T^{\prime} \cdot U$ with $T^{\prime}=S^{-1} \oplus S$ on $M \oplus M$ and $S \in G L(M)^{+}$(resp. $\left.S \in G L(M)_{2}^{+}\right)$.

(b) If $T \in S p(K)$ (resp. $T \in r S p(K)$ ) then there are $U_{1}, U_{2} \in U(H)$ such that $T=U_{1} \cdot T^{\prime} \cdot U_{2}$ with $T^{\prime}$ as above.

Proof. (a) $T=\Lambda^{-1} \cdot S^{-1} \cdot \Lambda \oplus S^{\prime}$ on $K=\Lambda^{-1} K_{2} \oplus K_{2}$, with $S^{\prime} \in G L\left(K_{2}\right)^{+}$. Also $\operatorname{dim} K_{2}=\operatorname{dim} M$. Let $U_{0}$ be an orthogonal map from $K_{2}$ onto $M$. Any 
$z \in K$ is $\Lambda^{-1} x \oplus y$ with $x, y \in K_{2}$. Let $U$ be defined by $U z=\Lambda^{-1} U_{0} x \oplus U_{0} y$. Then $U \in U(H)$. Let $S$ on $M$ be defined by $S=U_{0} S^{\prime} U_{0}^{-1}$. Then $S$ has the desired form and $U^{-1} \cdot T^{\prime} \cdot U=T$. Part (b) follows since $S p(K)$ and $r S p(K)$ are stable under polar decomposition.

The following lemma is needed in the proof of Theorem 4.2.

Lemma 2.4. Let $T \in S p(K)_{2}^{+}$with $T=S^{-1} \oplus S$ on $K=M \oplus M$. Suppose that each element in the spectrum of $T$ has multiplicity 1 and (hence) that 1 is not in the spectrum. If $T_{n} \rightarrow T$ in $S p(K)_{2}^{+}$then there is a sequence $T_{n}^{\prime}$ in $S p(K)_{2}^{+}$each having the same eigenvectors as $T$ and $U_{n} \in U(H)$ such that $T_{n}=U_{n} \cdot T_{n}^{\prime} \cdot U_{n}^{-1}$ with $U_{n} \rightarrow I$ in $U(H)$ and $T_{n}^{\prime} \rightarrow T$ in $S p(K)_{2}^{+}$.

Proof. Since $K$ must be separable $T$ (resp. $T_{n}$ ) has an eigensystem $\left\{\lambda_{i}, e_{i}\right\}$ (resp. $\left.\left\{\lambda_{i, n}, e_{i, n}\right\}\right)$ where $i$ ranges over the nonzero integers. It may be supposed that $\lambda_{-i}=\lambda_{i}^{-1}$ and $\Lambda e_{-i}=e_{i}$ (resp. $\lambda_{-i, n}=\lambda_{i, n}^{-1}$ and $\Lambda e_{-i, n}=e_{i, n}$ ) and further that $e_{i, n} \rightarrow e_{i}$ and $\lambda_{i, n} \rightarrow \lambda_{i}$ for all $i$. (Cf. [15, p. 58]). We have $\Lambda \cdot U_{n}$ $=U_{n} \cdot \Lambda$ and hence $U_{n} \in U(H)$. Also $U_{n} \rightarrow I$ in $U(H)$. Let $T_{n}^{\prime}=U_{n}^{-1} \cdot T_{n} \cdot U_{n}$. Then $T_{n}^{\prime} \rightarrow T$ in $r S p(K)$ and hence in $S p(K)_{2}^{+}$.

3. The action of $r G L(M)$ on $L_{2}(M, n)$. 3.1. A complex valued function $f(\cdot)$ on the real Hilbert space $M$ is tame if there is a subspace $M^{\prime}$ with $\operatorname{dim} M^{\prime}$ $=r$, finite, and a bounded Baire function $\bar{f}(\cdot)$ on $M^{\prime}$ such that $f(x)=\bar{f}(P x)$ where $P$ is the projection with range $M^{\prime}$. Let

$$
E(f)=\int(2 \pi c)^{-r / 2} \bar{f}(x) \cdot \exp (-(x, x) / 2 c) d x,
$$

the integral being taken over $M^{\prime}$ with regard to Lebesgue measure. There exists an essentially unique probability measure space $\Gamma=(N, \mathfrak{R}, n)$ and an identification map (the normal distribution over $M$ ) of the tame functions on $M$ to measurable functions on $N$ such that the $\sigma$-algebra determined by the tame functions is $\mathfrak{N}$ and $E(f)=\int f d n$ the integral being taken over $N$. (Cf. [7, p. 116 et seq.].) When a function on $N$ corresponds to a function on $M$ the distinction between them will be ignored. $I_{0}$ is the identity function on $N$. We write $L_{i}(M, n)$ instead of $L_{i}(\Gamma)$.

For $T \in G L(M)$ there is an automorphism $\phi(T)$ of $L_{\infty}(M, n)$ such that $\phi(T) f(x)=f\left(T^{*} x\right)$ if and only if $T \in r G L(M)$ [10, Theorem 3]. For $T \in r G L(M)$, let $n(T)$ be the measure defined on $\mathfrak{N}$ by $n(T)(S)=\int \phi^{-1}(T) \aleph_{s} d n$ where $S \in \mathfrak{N}$ and $\boldsymbol{\aleph}_{s}$ is the characteristic function of $S$. Let $X(T)$ denote the RadonNikodym derivative of $n(T)$ with regard to $n$. If $|T| \in G L(M)_{1}, X(T)$ $=\Delta(|T|) \exp \left(-\left(\left(T \cdot T^{*}-I\right) x, x\right) / 2 c\right) ;[10$, p. 23]. Let $\mathfrak{U}(\cdot)$ be the unitary representation of $r G L(M)$ on $L_{2}(M, n)$ which is defined on the tame functions by $\mathfrak{u}(T) f(x)=X(T)^{1 / 2} f\left(T^{*} x\right)$. If $M_{0}$ denotes the operation on $L_{2}(M, n)$ of multiplication by $g$ then for $g \in L_{\infty}(M, n), M_{\phi(T) \theta}=\mathfrak{u}(T) \cdot M_{\theta} \cdot \mathfrak{u}(T)^{-1}$.

THEOREM 3.1. $\mathfrak{U}(\cdot)$ is a weakly continuous unitary representation of $r G L(M)$ on $L_{2}(M, n)$. 
Theorem 3.1 will be used in the proof of Theorem 4.2, where a somewhat more special result would suffice. It is included because it seems to have some independent interest. The proof depends on the following lemmas.

LEMMA 3.1. There is a dense domain $D$ of tame functions in $L_{2}(M, n)$ such that if $\Phi(T)$ is defined by $\Phi(T) f(x)=f\left(T^{*} x\right)$ for all $T \in G L(M)$ and $f(\cdot) \in D$, then $T_{n} \rightarrow T$ strongly implies $\Phi\left(T_{n}\right) f \rightarrow \Phi(T) f$ in $L_{2}(M, n)$.

Proof. Let $D$ be the (algebraic) algebra of tame functionals in $L_{2}(M, n)$ generated by the functions of the form $r((\cdot, e))$, where $e$ is a unit vector in $M$ and $r(\cdot)$ is a continuous function on the reals with compact support. For fixed $e$, the functions $r((\cdot, e))$ are dense in $L_{2}\left(M^{\prime}, n\right)$ where $M^{\prime}$ is the subspace generated by $e$. Any subspace $M^{\prime \prime}$ of $M$, with finite dimension, is the direct sum of subspaces $M_{1}, \cdots, M_{n}$ each of dimension 1 and $L_{2}\left(M^{\prime \prime}, n\right)$ $=L_{2}\left(M_{1}, n\right) \otimes \cdots \otimes L_{2}\left(M_{n}, n\right)$. It follows that the closure of $D$ contains all tame functionals based on $M^{\prime \prime}$. Therefore $D$ is dense in $L_{2}(M, n)$. If $T_{n} \rightarrow T$ strongly then $T_{n} e \rightarrow T e$ in $M$. It follows that $\left(\cdot, T_{n} e\right) \rightarrow(\cdot, T e)$ in $L_{2}(M, n)$ and hence also in measure. Hence $r\left(\left(\cdot, T_{n} e\right)\right) \rightarrow r((\cdot, T e))$ in $L_{2}(M, n)$. The lemma follows readily.

LEMma 3.2. Let $T \in G L(M)_{2}^{+}$and $\left\{e_{\alpha}, \lambda_{\alpha}\right\}$ be an eigensystem for $T$. Then $\int X(T)^{1 / 2} d n=\prod_{\alpha}\left(2 \lambda_{\alpha} /\left(\lambda_{\alpha}^{2}+1\right)\right)^{1 / 2}$, where the integral is over $N$.

Proof. Let $\theta$ range over the collection of finite subsets of $\{\alpha\}$ and let these sets be directed by inclusion. Let $T_{\theta}$ be the operator determined by the equations: $T_{\theta} e_{\alpha}=\lambda_{\alpha} e_{\alpha}$ if $\alpha \in \theta, T_{\theta} e_{\alpha}=e_{\alpha}$ otherwise. It follows from [10, Lemma $1.1]$ that $X\left(T_{\theta}\right) \rightarrow X(T)$ in $L_{1}(M, n)$. The inequality $|a-b|^{2} \leqq\left|a^{2}-b^{2}\right|$, for positive $a$ and $b$, now implies that $\left(X\left(T_{\theta}\right)\right)^{1 / 2} \rightarrow(X(T))^{1 / 2}$ in $L_{2}(M, n)$. Since the functions are on a probability measure space, convergence is in $L_{2}(M, n)$ also. Using a formula given above for $X(T)$ when $T$ is in $G L(M)_{1}^{+}$, $\int\left(X\left(T_{\theta}\right)\right)^{1 / 2} d n$ may be evaluated as $\prod_{\alpha \in \theta}\left(2 \lambda_{\alpha} / \lambda_{\alpha}^{2}+1\right)^{1 / 2}$. The lemma follows.

Lemma 3.3. $X(\cdot)^{1 / 2}$, considered as a function from $G L(M)_{2}^{+}$to $L_{2}(M, n)$, is continuous at $I$.

Proof. $\left(X(I)^{1 / 2}\right)=I_{0}$. From Lemma 3.2 it follows with the notation used there that

$$
\begin{aligned}
\left\|X(T)^{1 / 2}-I_{0}\right\|^{2} & =2\left(1-\prod_{\alpha}\left(2 \lambda_{\alpha} / \lambda_{\alpha}^{2}+1\right)^{1 / 2}\right) \\
& =2\left(1-\prod_{\alpha}\left(1+\left(\delta_{\alpha}^{2} / 2\left(1+\delta_{\alpha}\right)\right)^{-1 / 2}\right)\right), \text { where } \lambda_{\alpha}=1+\delta_{\alpha} .
\end{aligned}
$$

Now $1 \leqq \prod_{\alpha}\left(1+\delta_{\alpha}^{2} / 2\left(1+\delta_{\alpha}\right)\right) \leqq \exp \left(\sum_{\alpha}\left(\delta_{\alpha}^{2} / 2\left(1+\delta_{\alpha}\right)\right)\right)$. Provided that $\|T-I\|_{2}<1 / 2$, it may be seen that $\exp \left(\sum_{\alpha}\left(\delta_{\alpha}^{2} / 2\left(1+\delta_{\alpha}\right)\right)\right) \leqq \exp \left(\|T-I\|_{2}^{2}\right)$. Hence $\exp \left(-\|T-I\|_{2}\right) \leqq \int X(T)^{1 / 2} \cdot d n \leqq 1$. The lemma follows.

Proof of Theorem 3.1. It is sufficient to establish weak continuity at $I$. Since $\mathfrak{U}(\cdot)$ restricted to $O(M)$ is weakly continuous, [7, Corollary 3.1$]$; it is 
enough to establish continuity on $G L(M)_{2}^{+}$. Taking $D$ as in Lemma 3.1 , for $f \in D$ and $T \in G L(M)_{2}^{+},\|\mathfrak{u}(T) f-f\|=\left\|X(T)^{1 / 2} \phi(T) f-f\right\| \leqq\left\|\left(X(T)^{1 / 2}-1\right) \phi(T) f\right\|$ $+\|\phi(T) f-f\|$. But $|\phi(T) f| \leqq \alpha$, with $\alpha$ independent of $T$. Therefore $\|\mathfrak{u}(T) f-f\| \leqq \alpha\left\|X(T)^{1 / 2}-I_{0}\right\|+\|\phi(T) f-f\|$. By Lemmas 3.1 and 3.3 this $\rightarrow 0$ as $T \rightarrow I$. This proves the theorem.

The Wiener transform $W$ is the unitary operator on $L_{2}(M, n)$ whose action on polynomials is given by $W f(x)=\int f\left(2^{1 / 2} y+i x\right) d n$ the integral being taken with regard to $y$; $\left[7\right.$, p. 119]. The map $T \rightarrow T^{*-1}$ is a continuous involution of $r G L(M)$ and hence $T \rightarrow \mathfrak{U}\left(T^{*-1}\right)$ is a weakly continuous representation.

Corollary 3.1.1. For $T \in r G L(M), W \cdot \mathfrak{u}(T) \cdot W^{-1}=\mathfrak{u}\left(T^{*-1}\right)$.

Proof. Since $G L(M)_{0}$ is dense in $r G L(M)$, by the theorem it is sufficient to establish the result for $T \in G L(M)_{0}$. Since $W$ commutes with $\mathfrak{U}(V)$ for $V \in O(M),\left[7\right.$, p. 125]; $T$ may be supposed in $G L(M)_{2}^{+}$. Hence it is enough to consider $T=\lambda I \oplus I$ on $M_{1} \oplus M_{1}^{\perp}$ with $\operatorname{dim} M_{1}=1$, or equivalently to consider the case when $\operatorname{dim} M=1$. But then the unitary map $f(x) \rightarrow(2 \pi c)^{-1 / 4}$ $\cdot \exp \left(-x^{2} / 4 c\right) f(x)$ from $L_{2}(M, n)$ to the square integrable functions with regard to Lebesgue measure, transforms $W$ into the Fourier transform $F,[7$, p. 122]; and $\mathfrak{u}(\lambda I) f(x)$ is now $|\lambda|{ }^{1 / 2} f(\lambda x)$. An elementary computation gives $F \cdot \mathfrak{u}(\lambda I)=\mathfrak{u}\left(\lambda^{-1} I\right) \cdot F$.

REMARK 3.1. The Jacobian $X(T)$ is the analogue of $|\Delta(T)|$ when $\operatorname{dim} M$ is finite and the normal distribution is replaced by Lebesgue measure. We have:

CoROllaRy 3.1.2. $X(\cdot)$ is a continuous function from $G L(M)_{2}$ to $L_{1}(M, n)$.

Proof. Since $X(T)^{1 / 2}=\mathfrak{u}(T) I_{0}$, by the theorem, $T \rightarrow X(T)^{1 / 2}$ is continuous to $L_{2}(M, n)$. Also $\int X(T) d n=1$. If $f_{n}$ is a sequence of non-negative functions on a probability measure space, each with expectation 1 , and $f_{n}^{1 / 2} \rightarrow f^{1 / 2}$ in mean, it may be seen that $f_{n} \rightarrow f$ in $L_{1}$. The corollary follows.

4. The unitary action of $r S p(K)$ on the Fock-Cook field operators. 4.1. Let $H$ be a complex Hilbert space of arbitrary dimension, $H_{0}$ be a complex Hilbert space of dimension 1 with unit vector $e_{0}, H_{n}=H \otimes \cdots \otimes H$ ( $n$ times) and $F=\oplus \sum_{n=0}^{\infty} H_{n}$. For $x_{1}, \cdots, x_{n} \in H$ (not necessarily 'distinct, and $n \geqq 1$ ) the symmetric tensor product $\left(x_{1} \otimes \cdots \otimes x_{n}\right)_{s}=(n !)^{-1} \sum x_{\pi(1)} \otimes \cdots \otimes x_{\pi(n)}$, where summation is over all permutations $\pi(\cdot)$ of $1, \cdots, n$. The symmetric tensor algebra $S(H)_{0}$ is the linear manifold in $F$ generated by $e_{0}$ and all $\left(x_{1} \otimes \cdots \otimes x_{n}\right)_{s}, n=1,2,3, \cdots$. The space of symmetric tensors $S(H)$ is the closure of $S(H)_{0}$. Let $C_{0}(z)$ be defined on $S(H)_{0}$ by $C_{0}(z) e_{0}=z$, $C_{0}(z)\left(x_{1} \otimes \cdots \otimes x_{n}\right)_{s}=(n+1)^{1 / 2}\left(z \otimes x_{1} \otimes \cdots \otimes x_{n}\right)_{s}$ and $C_{0}^{*}(z)$ be the adjoint of $C_{0}(z)$ restricted to $S(H)_{0}$. The Fock-Cook quantization on $S(H), R(\cdot)$, is given by $R(z)=\left(1 / 2^{1 / 2}\right)\left(C_{0}(z)+C_{0}^{*}(z)\right)^{\sim}$ where $\sim$ indicates closure [3]. Let $M$ be the real subspace of $K$ defined in $\S 2$. Let $D$ be the duality transform 
from $S(H)$ to $L_{2}(M, n)$. (See $[7$, p. 123].) The Fock-Cook quantization on $L_{2}(M, n)$ is defined by $R(z)=D \cdot\left(C_{0}(z)+C_{0}^{*}(z)\right)^{\sim} \cdot D^{-1}$. Then decomposing $K$ as $M \oplus M$ and for $x \in M$ writing $P(x)=R(x \oplus 0)$ and $Q(x)=R(0 \oplus-x)$ we have $Q(x)=M((\cdot, x) / 2 c)$ on $L_{2}(M, n)$ where $M(g)$ indicates the operation of multiplication by $g$, and $P(x)=W \cdot Q(x) \cdot W^{-1}[7$, p. 126].

The canonical unitary representation $\gamma(\cdot)$ of $U(H)$ on $S(H)$ is determined by: $\gamma(U) e_{0}=e_{0}, \gamma(U)\left(x_{1} \otimes \cdots \otimes x_{n}\right)_{s}=\left(U x_{1} \otimes \cdots \otimes U x_{n}\right)_{s}$. When $U(H)$ is given the weak operator topology $\gamma(\cdot)$ is weakly continuous. $\Gamma(\cdot)$ $=D \cdot \gamma(\cdot) \cdot D^{-1}$.

Definition 4.1. If $T \in r S p(K)$ and $T=U_{1} \cdot T^{\prime} \cdot U_{2}$ (decomposition of Lemma 2.3) then $Y(T)=\Gamma\left(U_{1}\right) \cdot \mathfrak{u}(S) \cdot \Gamma\left(U_{2}\right)$ and $\bar{Y}(T)$ is the unitary ray $\{\alpha Y(T):|\alpha|=1\}$

TheOREM 4.1. Let $T \in S p(K)$ and $R(\cdot)$ be the Fock-Cook quantization on $L_{2}(M, n)$. There is a unitary operator $X$ such that $R(T z)=X \cdot R(z) \cdot X^{-1}$ for all $z \in K$ if and only if $T \in r S p(K)$ and then $X \in \bar{Y}(T) . Y(T)$ is characterised by $\left(Y(T) I_{0}, I_{0}\right)>0$. Finally $\bar{Y}(\cdot)$ is a projective unitary representation of $r S p(K)$.

Proof. By considering the representation $\Gamma(\cdot)$ of $U(H)$ and Lemma 2.3 we see that to establish the first part $T$ may be supposed to be of the form $S^{-1} \oplus S$ on $M \oplus M$ with $S$ positive selfadjoint. Suppose $X \cdot R(z) \cdot X^{-1}=R(T z)$ for all $z \in K$. Then $X \cdot Q(x) \cdot X^{-1}=Q(S x)$ for all $x \in M$. Hence for $g \in L_{\infty}(M, n)$, $M(g) \rightarrow X \cdot M(g) \cdot X^{-1}$ determines an automorphism on $L_{\infty}(M, n)$ which takes $(\cdot, x)$ into $(\cdot, S x)$. Therefore by $[10$, Theorem 3] $S \in r G L(M)$ and hence $T \in r S p(K)$. Now suppose $T \in r S p(K)$ then $Y(T)=\mathfrak{u}(S), Y(T) \cdot Q(x) \cdot Y(T)^{-1}$ $=Q(S x)$ and $Y(T) \cdot P(x) \cdot Y(T)^{-1}=\mathfrak{u}(S) \cdot W \cdot Q(x) \cdot W^{-1} \cdot \mathfrak{u}(S)^{-1}=W \cdot \mathfrak{u}\left(S^{-1}\right)$ $\cdot Q(x) \cdot \mathfrak{u}\left(S^{-1}\right)^{-1} \cdot W^{-1}$ (by Corollary 3.1 .1$)=W \cdot Q\left(S^{-1} x\right) \cdot W^{-1}=P\left(S^{-1} x\right)$. Now decomposing $z \in K$ as $x \oplus y$ on $M \oplus M$ we have $R(z)=(P(x)+Q(-y))^{\sim}$. It follows that $Y(T) \cdot R(z) \cdot Y(T)^{-1}=R(T z)$. Since the Fock-Cook quantization is irreducible, $[3,7]$; it follows that any two unitary operators transforming $R(z)$ into $R(T z)$, belong to the same unitary ray and that $\bar{Y}(\cdot)$ is a projective unitary representation. Finally let $T \in r S p(K)$ and $T=U_{1} \cdot T^{\prime} \cdot U_{2}$ (decomposition of Lemma 2.3). Then $\left(Y(T) I_{0}, I_{0}\right)=\left(\mathfrak{U}(S) I_{0}, I_{0}\right)$ (since $\Gamma(\cdot)$ leaves $I_{0}$ fixed $)=\int X(S)^{1 / 2} d n>0$, the integral being taken over $N$. A unitary ray can have at most one element with this property. Thus the theorem is proved.

A projective unitary representation $\bar{Z}(\cdot)$ of a topological group $G$ is continuous if $T \rightarrow|(\bar{Z}(T) x, y)|$ is continuous for each pair of vectors $x, y$.

THEOREM 4.2. $\bar{Y}(\cdot)$ is a continuous projective unitary representation.

We establish this by showing that $Y(\cdot)$ is weakly continuous.

Proof of Theorem. Since $Y(T)=\Gamma(u(T)) \cdot Y(|T|)$ and $\Gamma(\cdot)$ is weakly continuous it is sufficient to establish the weak continuity of $Y(\cdot)$ on $S p(K)_{2}^{+}$. Let $T_{n} \rightarrow T$ in $S p(K)_{2}^{+}$. By Lemma 2.3 there is no loss in supposing that $T$ 
decomposes as $S^{-1} \oplus S$ on $M \oplus M$. Since $T-I$ and $T_{n}-I$ are Hilbert-Schmidt there is a separable subspace $K^{\prime}$ of $K$ such that $T$ and each $T_{n}$ are reduced by $K^{\prime}$ and are $I$ on $K^{\prime \perp}$. $K^{\prime}$ may be supposed to be determined by a complex subspace $H^{\prime}$ of $H$. On decomposing $H$ as $H^{\prime} \oplus H^{\prime \perp}$, and $K$ as $K^{\prime} \oplus K^{\prime \perp}, M$ becomes $M^{\prime} \oplus M^{\prime \perp}$ (orthogonal complement in $M$ ), and $L_{2}(M, n)$ $=L_{2}\left(M^{\prime}, n\right) \otimes L_{2}\left(M^{\prime \perp}, n\right)$. If $T=T^{\prime} \oplus T^{\prime \prime}$ on $K^{\prime} \oplus K^{\prime \perp}$ then $Y(T)$ $=Y\left(T^{\prime}\right) \otimes Y\left(T^{\prime \prime}\right)$. Consequently in establishing continuity there is no loss in supposing $K$ is separable. Suppose $T$ satisfies the hypothesis of Lemma 2.4. Then adopting the notation of the lemma, since $T_{n}^{\prime}$ can be written as $S_{n}^{-1} \oplus S_{n}$ with $S_{n} \rightarrow S$ in $G L(M)_{2}$, we have, by Theorem 3.1, $Y\left(T_{n}\right)=\Gamma\left(U_{n}\right) \cdot \mathfrak{u}\left(S_{n}\right)$ $\cdot \Gamma\left(U_{n}^{-1}\right) \rightarrow \mathfrak{U}(S)=Y(T)$ (weak convergence). In the general case there is a selfadjoint operator $A \in r S p(K)$ which commutes with $T$ such that $A \cdot T$ satisfies the conditions of the lemma. Then $Y\left(\left|A \cdot T_{n}\right|\right) \rightarrow Y(A \cdot T)$ weakly. Since $u\left(A \cdot T_{n}\right) \rightarrow I$ weakly $\Gamma\left(u\left(A \cdot T_{n}\right)\right) \rightarrow I$ weakly, and $Y\left(\left|A \cdot T_{n}\right|\right)$ $=\Gamma\left(u\left(A \cdot T_{n}\right)\right) \cdot Y\left(\left|A \cdot T_{n}\right|\right) \rightarrow Y(A \cdot T)=Y(A) \cdot Y(T)$ weakly. Hence on multiplying through by $Y(A)^{-1}$, there are $X_{n} \in \bar{Y}\left(T_{n}\right)$ such that $X_{n} \rightarrow Y(T)$ weakly. Hence $Y\left(T_{n}\right) \rightarrow Y(T)$ weakly. This completes the proof.

REMARK 4.1. The Hilbert space $K$ plays to some extent the role of phase space in classical mechanics. Let $\left\{e_{\alpha}\right\}$ be an orthonormal basis for $M$. For $z \in K=M \oplus M$ we write $z=\sum_{\alpha} p_{\alpha} e_{\alpha} \oplus q_{\alpha} e_{\alpha}$. Let $h\left(p_{\alpha}, q_{\alpha}\right)$ be a real quadratic form in the $p_{\alpha}$ and $q_{\alpha}$ without linear terms. The correspondence principle for determining the field operator corresponding to the classical Hamiltonian $h\left(p_{\alpha}, q_{\alpha}\right)$ amounts to replacing $p_{\alpha}$ by $P_{\alpha}=P\left(e_{\alpha}\right), q_{\alpha}$ by $Q_{\alpha}=Q\left(e_{\alpha}\right)$ and $h\left(p_{\alpha}, q_{\alpha}\right)$ by $h\left(P_{\alpha}, Q_{\alpha}\right)$. The difficulty is that $h\left(P_{\alpha}, Q_{\alpha}\right)$ may be nowhere defined. One cause is that $h\left(P_{\alpha}, Q_{\alpha}\right)$ differs from a selfadjoint operator by a physically irrelevant "infinite constant." An alternative method of quantization equivalent to the above for systems with finitely many degrees of freedom is to find the 1-parameter subgroup $\theta(t)$ of $S p(K)$, (provided it exists) which is determined by the classical equations of motion and Hamiltonian $h\left(p_{\alpha}, q_{\alpha}\right)$. It follows from Theorems 4.1 and 4.2 (by choosing a 1-parameter unitary group from $\bar{Y}(\theta(t))$ and applying Stone's Theorem) that $\theta(t)$ determines a selfadjoint operator on $L_{2}(M, n)$ if and only if $\theta(t)$ is a continuous subgroup of $r S p(K)$.

Consider the Hamiltonian $h(p, q)=\sum a_{\alpha} p_{\alpha}^{2}$. It is shown in [8, p. 302] that $\sum_{\alpha} a_{\alpha} P_{\alpha}^{2}$ is a selfadjoint operator if and only if $\sum_{\alpha}\left|a_{\alpha}\right|<\infty$. However $\theta(t)\left(p_{\alpha} e_{\alpha} \oplus q_{\alpha} e_{\alpha}\right)=p_{\alpha} e_{\alpha} \oplus\left(2 a_{\alpha} t p_{\alpha}+q_{\alpha}\right) e_{\alpha}$. It may be seen $\theta(t)$ is a continuous subgroup of $r S p(K)$ if and only if $\sum_{\alpha} a_{\alpha}^{2}<\infty$. Thus although the divergence may be said to be due to an "infinite constant" if $\sum_{\alpha} a_{\alpha}^{2}$ converges, it seems in the general case that there is no way to obtain a selfadjoint operator in $L_{2}(M, n)$.

4.2. A tame function $f(x)$ on $M$ is even (resp. odd) if $f(-x)=f(x)$ (resp. $f(x)=-f(x)$ ). Let $L^{+}$(resp. $L^{-}$) be the closure in $L_{2}(M, n)$ of the linear manifold of even (resp. odd) tame functions. $L_{2}(M, n)=L^{+} \oplus L^{-}$. Let $L_{n}$ be 
the image under the duality transform of the subspace of $S(H)$ generated by the symmetric $n$-tensors. Then $L^{+}=\oplus \sum_{n=0}^{\infty} L_{2 n}$ and $L^{-}=\oplus \sum_{n=0}^{\infty} L_{2 n+1}$. It may be seen that $\Gamma(-I)=I \oplus-I$ on $L^{+} \oplus L^{-}$and $\Gamma(-I) \cdot Y(T)=Y(T)$ $\cdot \Gamma(-I)$ for all $T \in r S p(K)$. Therefore $L^{+}$and $L^{-}$are invariant under all $\bar{Y}(T)$.

THEOREM 4.3. The subspaces $L^{+}$and $L^{-}$are irreducible under $\bar{Y}(\cdot)$ restricted to $S p(K)_{0}$.

Proof. The subspaces $L_{n}$ are invariant and irreducible under $\Gamma(\cdot)$, [9]. Since $\Gamma(\cdot)$ is weakly continuous and $U\left(H_{0}\right)$ is weakly dense in $U(H)$ it follows that the $L_{n}$ are irreducible under $\Gamma(\cdot)$ restricted to $U(H)_{0}$. Let $e$ be a unit vector in $M$. Then $I_{0} \in L^{+}$and $(\cdot, e) \in L^{-}$. Let $S(\lambda): S(\lambda) e=\lambda e$ and $S(\lambda) e^{\prime}=e^{\prime}$ for $e^{\prime} \perp e$. It is sufficient to show that the subspace generated by the linear manifold $V^{+}$(resp. $V^{-}$) determined by $\left\{\mathfrak{u}(S(\lambda)) I_{0}: \lambda>1\right\}$ (resp. by $\{\mathfrak{U}(S(\lambda))(\cdot, e): \lambda>1\})$ has nonempty intersection with $L_{n}$ for $n$ even (resp. $n$ odd). In establishing this there is no loss in assuming that $M$ is the 1 dimensional space generated by $e$. Identifying $M$ with the real line we have $\mathfrak{U}(S(\lambda)) f(x)=\lambda^{1 / 2} \exp \left(-\left(\lambda^{2}-1\right) x^{2} / 4 c\right) f(\lambda x)$. (See §3.) Then $V^{+}$is the manifold generated by $\left\{\exp \left(-\delta x^{2}\right): \delta>0\right\} . V^{+}$is closed under multiplication. An application of the Stone-Weierstrass theorem shows that $V^{+}$is uniformly dense in the even continuous functions vanishing at infinity and hence the $L_{2}$ closure of $V^{+}$is $L^{+} . V^{-}$is generated by $\left\{x \cdot \exp \left(-\delta x^{2}\right): \delta>0\right\}$. Suppose $f(x) \in L^{-}$and $f(x) \perp V^{-}$. Then $x \cdot \exp \left(-x^{2}\right) \cdot f(x) \in L^{+}$and is orthogonal to $V^{+}$. Therefore it is zero a.e. Therefore $f(x)=0$ a.e. Therefore $V^{-}$is dense in $L^{-}$.

5. The analogue of the spinor representation of the orthogonal group. 5.1. We now suppose that $\operatorname{dim} H$ is finite. Then $r S p(K)=S p(K)$. For $T \in S p(K)$ let $\delta(T)=\Delta(u(T))$ where $\Delta(\cdot)$ is the complex determinant on $U(H)$.

THEOREM 5.1. The unitary ray representation of $S p(K), \bar{Y}(\cdot)$, determines uniquely the double valued unitary representation $\delta(\cdot)^{1 / 2} Y(\cdot)$.

Proof. Since $Y(\cdot)$, although given explicitly is difficult to treat, the proof is indirect. $S p(K)$ is a semisimple Lie group with Lie algebra $\mathfrak{g}=\left\{X\right.$ on $\left.K: \Lambda \cdot X+X^{*} \cdot \Lambda=0\right\}$, supplied with the usual bracket operation. The function $\exp (\cdot)$ maps the selfadjoint elements of $g$ homeomorphically onto $S p(K)^{+}$, which consequently is simply connected. Let $\bar{S} p(K)$ be the universal covering group of $S p(K)$ and $p$ the canonical homomorphism onto $S p(K)$. Then $\bar{U}(H)=p^{-1}(U(H))$, is the universal covering group of $U(H)$. Let $\bar{S} p(K)^{+}$be the component of $p^{-1}\left(S p(K)^{+}\right)$which contains the identity element $\bar{I}$. Then $\bar{S} p(K)^{+}$is homeomorphic to $S p(K)^{+}$under $p$. Any $\bar{T} \in \bar{S} p(K)$ can be decomposed uniquely as $\bar{U} \cdot \bar{T}_{1}$ with $\bar{U} \in \bar{U}(H)$ and $\bar{T}_{1} \in \bar{S} p(K)^{+}$. The proofs of the above are simple and are omitted.

Since $S_{p}(K)$ is semisimple, by Theorem 4.2 and [1, Theorem 7.1$]$, there 
is a neighbourhood $N$ of $I$ and a continuous function $\sigma(\cdot)$ on $N$ such that $T \rightarrow \sigma(T) Y(T)$ is a local unitary representation. We may suppose that $N$ is an admissible neighbourhood for $p$. Let $\bar{N}$ be the component of $p^{-1}(N)$ which contains $\bar{I}$. The local representation extends uniquely to a representation $Z(\cdot)$ of $\bar{S} p(K) . Z(\cdot)$ must have the form $Z(\bar{T})=\bar{\sigma}(\bar{T}) Y(p \bar{T})$, where $\bar{\sigma}(\cdot)$ is a continuous function on $\bar{S} p(K)$ corresponding to $\sigma(\cdot)$ on $\bar{N}$. Since $Y(\cdot)$ is the representation $\Gamma(\cdot)$ on $U(H), \bar{\sigma}(\cdot)$ is a 1-dimensional representation of $\bar{U}(H)$. It follows that on $\bar{U}(H), \bar{\sigma}(\bar{U})=\Delta(p \bar{U})^{\alpha}$ with $\alpha$ real.

We next show that $\bar{\sigma}(\cdot)=1$ on $\bar{S} p(K)^{+}$. Let $e_{1}, \cdots, e_{n}$ be an orthonormal basis for $M$. For $i=1, \cdots, n$, let $T_{i}(t)=S_{i}^{-1}(t) \oplus S_{i}(t)$ on $K=M \oplus M$, where $S_{i}(t) e_{j}=\exp \left(\delta_{i j} t\right) e_{j}$ for $j=1, \cdots, n$. The $T_{i}(t)$, (all $i$, all $t$ ) generate an abelian subgroup $G$ of $S p(K)$. By Lemma 2.3, if $T \in S p(K)^{+}$then $T=U^{-1} \cdot T^{\prime} \cdot U$ with $U \in U(H)$ and $T^{\prime} \in G$. If $\bar{T}, \bar{T}^{\prime}$ are the corresponding elements in $\bar{S} p(K)^{+}$ and $p \bar{U}=U$, then $\bar{T}=\bar{U}^{-1} \cdot \bar{T}^{\prime} \cdot \bar{U}$. Since $\left(Y(U)^{-1} \cdot Y\left(T^{\prime}\right) \cdot Y(U) I_{0}, \quad I_{0}\right)$ $=\left(Y\left(T^{\prime}\right) \Gamma(U) I_{0}, \Gamma(U) I_{0}\right)=\left(Y\left(T^{\prime}\right) I_{0}, I_{0}\right)>0$, it follows by Theorem 4.1 that $Y(T)=Y(U)^{-1} \cdot Y\left(T^{\prime}\right) \cdot Y(U)$. It follows that $\bar{\sigma}(T)=\bar{\sigma}\left(T^{\prime}\right)$. Again $Y(\cdot)$ may be seen to be multiplicative on $G$ (cf. Theorem 3.1). Hence it is sufficient to consider there 1-parameter groups $\bar{T}_{i}(t)$ where $\bar{\sigma}(\cdot)$ must have the form $\bar{\sigma}\left(\bar{T}_{i}(t)\right)=\exp \left(i \beta_{i} t\right)$ with $\beta_{i}$ real. But $\Lambda \cdot T_{i}(t) \cdot \Lambda^{-1}=T_{i}(-t)$. Hence if $p \bar{\Lambda}=\Lambda$, $\bar{\Delta} \cdot \bar{T}_{i}(t) \cdot \bar{\Lambda}^{-1}=\bar{T}_{i}(-t)$. Therefore $\exp \left(i \beta_{i} t\right)=\exp \left(-i \beta_{i} t\right)$ for all $t$. Therefore $\beta_{i}=0$. We have shown that $Z(\bar{T})=\delta(p \bar{T})^{\alpha} Y(p \bar{T})$. Let $K_{1}$ be the subspace of $K$ generated by $e_{1}$ and $\Lambda^{-1} e_{1}$. On embedding $S p\left(K_{1}\right)$ in $S p(K)$ in the natural way, and using the uniqueness of $Z(\cdot)$, it may be seen that $\alpha$ does not depend on the dimension of $H$.

We obtain $\alpha$ by analysing the case $\operatorname{dim} H=1$. Let $e$ be a unit vector in $M$. Then $e$ and $\Lambda^{-1} e$ are a basis for $K . S p(K)$ is the $2 \times 2$ real unimodular group. Its Lie algebra $g$ is all $2 \times 2$ real matrices with trace zero. The matrices

$$
X_{0}=\left(\begin{array}{rr}
0 & 1 \\
-1 & 0
\end{array}\right), \quad X_{1}=\left(\begin{array}{rr}
-1 & 0 \\
0 & 1
\end{array}\right), \quad \text { and } \quad X_{2}=\left(\begin{array}{ll}
0 & 1 \\
1 & 0
\end{array}\right)
$$

are a basis for g. We have $\left[X_{0}, X_{1}\right]=2 X_{2},\left[X_{1}, X_{2}\right]=-2 X_{0}$ and $\left[X_{2}\right.$, $\left.X_{0}\right]=2 X_{1}$. For all real $t$ and $i=0,1,2$, let $V_{i}(t)=\exp \left(t X_{i}\right)$. Then

$$
V_{0}(t)=\left(\begin{array}{cc}
\cos t & \sin t \\
-\sin t & \cos t
\end{array}\right), \quad V_{1}(t)=\left(\begin{array}{cc}
\exp (-t) & 0 \\
0 & \exp (t)
\end{array}\right)
$$

and $V_{2}(t)=V_{0}(\pi / 4) \cdot V_{1}(t) \cdot V_{0}(-\pi / 4)$. We note also that $V_{0}(t)=\exp (i t)$ on $H$.

We identify $L_{2}(M, n)$ with $L_{2}(R),(R=$ real numbers with Lebesgue measure) by the unitary map $f((x e, e)) \rightarrow(2 \pi c)^{-1 / 4} f(x) \exp \left(-x^{2} / 4 c\right)$. The spaces $L_{n}$, for $n=0,1,2, \cdots \quad$ (see $\S 4.2$ ) all have $\operatorname{dim} 1$. In $L_{2}(R), L_{n}$ is generated by the $n$th normalized hermite function $\psi_{n}=(\pi)^{-1 / 4}\left(2^{n} \cdot n !\right)^{-1 / 2} h_{n} \exp \left(-x^{2} / 2\right)$, where $h_{n}$ is the $n$th hermite polynomial and $n=0,1,2, \cdots,[7$, p. 124]. On $L_{2}(R)$ we have $\Gamma\left(V_{0}(T)\right) \psi_{n}=\exp ($ int $) \psi_{n}$ and $\mathfrak{U}(\lambda I) f(x)=\lambda^{1 / 2} f(\lambda x)$ for $\lambda>0$. Consequently $Y\left(V_{1}(t)\right) f(x)=\exp (t / 2) f(\exp (t) x)$. 
Following Harish-Chandra $f \in L_{2}(R)$ is well behaved for the representation $Z(\cdot)$ of $\bar{S} p(K)$ if $Z(\bar{T}) f$ is analytic at $\bar{I}\left[5\right.$, Part II]. Let $L^{0}$ be the (nonclosed) linear manifold generated by $\psi_{n}$ for all $n$. We show that $L^{0}$ is a linear manifold of well behaved vectors. Let $L^{0+}$ (resp. $L^{0-}$ ) be the submanifold generated by the $\psi_{n}$ for $n$ even(resp. $n$ odd). A compact form $g_{c}$ of the Lie algebra $\mathfrak{g}$ has basis $X_{0}, i X_{1}, i X_{2}$. Hence $\mathfrak{g} \cap \mathfrak{g}_{c}=\left\{X_{0}\right\}$. Therefore $\bar{U}(H)$ is the pseudo-compact subgroup of $\bar{S} p(K)$. The irreducible subspaces of $Z(\cdot)$ restricted to $\bar{U}(H)$ are the 1 -dimensional subspaces generated by the $\psi_{n}$. Since $S p(K)$ is semisimple and by Theorem $4.3 Z(\cdot)$ is irreducible on $L^{+}$and $L^{-}$, it follows from [5, Theorem 6], that $L^{0+}$ and $L^{0-}$ (and hence $L^{0}$ ) are linear manifolds of well behaved vectors.

For $X \in \mathfrak{g}$, let $d Z(X)$ be the infinitesimal generator of $Z(\exp (t X))$. Again by [5, Part II] $d Z(\cdot)$, restricted to the manifold of well behaved vectors, is a representation of $g$. We obtain $\alpha$ by computing $d Z(\cdot)$ explicitly on $L^{0}$.

Let $Y_{i}$ be the infinitesimal generator of the 1-parameter unitary group $Y\left(V_{i}(t)\right)$, for $i=0,1,2$. Then $d Z\left(X_{0}\right)=Y_{0}+i \alpha I, d Z\left(X_{1}\right)=Y_{1}$ and $d Z\left(X_{2}\right)=Y_{2}$. We have

$$
Y_{0} \psi_{n}=i n \psi_{n} .
$$

For $f(x) \in L^{0}$, since $Y\left(V_{1}(t)\right) f(x)=\exp (t / 2) \cdot f(\exp (t) \cdot x), \quad Y_{1} f(x)=(1 / 2) f(x)$ $+x \cdot d f(x) / d x$. On using $d\left(h_{n}\right) / d x=2 x \cdot h_{n}-h_{n+1}[18$, p. 54]; we have $Y_{1}\left(h_{n} \cdot \exp \left(-x^{2} / 2\right)\right)=\left(\left(x^{2}+1 / 2\right) h_{n}-x h_{n+1}\right) \cdot \exp \left(-x^{2} / 2\right)$. By $[18$, p. 52] $h_{n}=2^{n}\left(x^{n}-(n \cdot(n-1) / 4) x^{n-2}+(n \cdot(n-1) \cdot(n-2) \cdot(n-3) / 4.8) x^{n-4}-\cdots\right)$. An elementary (if tedious) computation gives: $h_{n+2}-4 x \cdot h_{n+1}+\left(4 x^{2}+2\right) \cdot h_{n}$ $-4 n(n-1) \cdot h_{n-2}=0$, where $h_{n}=0$ if $n<0$. Hence $Y_{1}\left(h_{n} \cdot \exp \left(-x^{2} / 2\right)\right)$ $=\left(-h_{n+2} / 4+n(n-1) h_{n-2}\right) \cdot \exp \left(-x^{2} / 2\right)$ or equivalently,

$$
Y_{1} \psi_{n}=-(1 / 2)((n+2) \cdot(n+1))^{1 / 2} \psi_{n+2}+(1 / 2)(n \cdot(n-1))^{1 / 2} \psi_{n-2} .
$$

It may be seen that $Y_{2}=\Gamma\left(V_{0}(\pi / 4)\right) \cdot Y_{1} \cdot \Gamma\left(V_{0}(-\pi / 4)\right)$. Hence

$$
X_{2} \psi_{n}=-i / 2((n+2) \cdot(n+1))^{1 / 2} \psi_{n+2}-i / 2(n \cdot(n-1))^{1 / 2} \psi_{n-2} .
$$

By (5.2) and (5.3) we have $\left[Y_{1}, Y_{2}\right] \psi_{n}=-2\left(Y_{0}+(1 / 2) i I\right) \psi_{n}$. But on $L^{0}$, $\left[Y_{1}, Y_{2}\right]=\left[d Z\left(X_{1}\right), d Z\left(X_{2}\right)\right]=-2 d Z\left(X_{0}\right)=-2\left(Y_{0}+i \alpha I\right)$. Therefore $\alpha=1 / 2$. This proves the theorem.

5.2. Now let $\operatorname{dim} H$ be infinite. If $M^{\prime}$ is a subspace of $M$ and $H^{\prime}$, and $K^{\prime}$ are the corresponding subspaces of $H$ and $K$, then if $T \in S p(K)$ is $T^{\prime} \oplus I$ on $K^{\prime} \oplus K^{\prime \perp}$, we have $Y(T)=Y\left(T^{\prime}\right) \otimes I$ on $L_{2}\left(M^{\prime}, n\right) \otimes L_{2}\left(M^{\prime}, n\right)$. It follows $\delta(\cdot)^{1 / 2} Y(\cdot)$ is a double valued unitary representation of $S p(K)_{0}$. Since the topology of $S p(K)_{1}$ is stronger than that derived from $r S p(K)$, it follows from Lemma 2.1 (a) and Theorem 4.2 that the representation extends to be continuous on $S p(K)_{1}$. However due to the nonexistence of even a local determinant on $U(H)_{2}$ (Lemma 2.1 (b)), the continuous unitary ray representation $\bar{Y}(\cdot)$ of $S p(K)_{2}$ cannot be smoothed to a unitary representation even locally. 
For $T, T^{\prime} \in r S p(K)$, let $Y(T) \cdot Y\left(T^{\prime}\right)=y\left(T, T^{\prime}\right) Y\left(T \cdot T^{\prime}\right)$. We have:

CoROllaRY 5.1.1. $y\left(T, T^{\prime}\right)^{2}=\Delta\left(u\left(u\left(T^{\prime}\right)^{-1} \cdot|T| \cdot u\left(T^{\prime}\right) \cdot\left|T^{\prime}\right|\right)\right)$, where $\Delta(\cdot)$ is the determinant on $U(H)_{1}$.

Lemma 5.1. $\left(T, T^{\prime}\right) \rightarrow u\left(T \cdot T^{\prime}\right)$ is a continuous function from $S p(K)_{2}^{+}$ $\times S p(K)_{2}^{+}$to $U(H)_{1}$.

Proof. Let $T=I+X, T^{\prime}=I+X^{\prime}, u\left(T \cdot T^{\prime}\right)=I+Y$ and $\left|T \cdot T^{\prime}\right|=I+Z$. Notice that $X, X^{\prime}$ and $Z$ are selfadjoint operators and that $X, X^{\prime}, Y$ and $Z$ are Hilbert-Schmidt. Denote $\left(C+C^{*}\right) / 2$ and $i\left(C^{*}-C\right) / 2$ by real $(C)$ and $\operatorname{Im}(C)$ respectively. We have $\operatorname{Im}\left(T \cdot T^{\prime}\right)=i\left(X^{\prime} \cdot X-X \cdot X^{\prime}\right) / 2$ and $\operatorname{Im}\left(u\left(T \cdot T^{\prime}\right)\right.$ $\left.\cdot\left|T, \quad T^{\prime}\right|\right)=\operatorname{Im}(I+Y+Z+Y \cdot Z)=i\left(Y^{*}-Y\right) / 2+i\left(Z \cdot Y^{*}-Y \cdot Z\right) / 2$. Hence $\operatorname{Im}\left(u\left(T \cdot T^{\prime}\right)\right)=\operatorname{Im}(I+Y)=i\left(X^{\prime} \cdot X-X \cdot X^{\prime}\right) / 2-i(Z \cdot Y-Y \cdot Z) / 2$, which is of trace class. Since $(I+Y) \cdot\left(I+Y^{*}\right)=I, \operatorname{real}\left(u\left(T \cdot T^{\prime}\right)\right)-I=\operatorname{real}(I+Y)-I$ $=\left(Y^{*}+Y\right) / 2=-Y \cdot Y^{*} / 2$, which is again of trace class. It follows that $u\left(T \cdot T^{\prime}\right)-I$ is of trace class. Therefore $u\left(T \cdot T^{\prime}\right) \in U(H)_{1}$. The proof of continuity follows readily and is omitted.

Proof of Corollary 5.1.1. Let $T, T^{\prime} \in r S p(K)$. Then $A=u\left(T^{\prime}\right)^{-1} \cdot|T| \cdot u\left(T^{\prime}\right)$ and $B=\left|T^{\prime}\right| \in S p(K)_{2}^{+}$. We have $T \cdot T^{\prime}=u(T) \cdot u\left(T^{\prime}\right) \cdot A \cdot B$. It follows that $Y\left(T \cdot T^{\prime}\right)=\Gamma\left(u(T) \cdot u\left(T^{\prime}\right)\right) \cdot Y(A \cdot B)$, and hence $y\left(T, T^{\prime}\right)=y(A, B)$. If $A, B$ $\in S p(K)_{0}$ it follows from Theorem 5.1 that $y(A, B)^{2}=\Delta(u(A \cdot B))$. In the general case let $A_{n}, B_{n} \in S p(K)_{0}^{+}$and $A_{n} \rightarrow A$ and $B_{n} \rightarrow B$ in $S p(K)_{2}^{+}$. By Lemma $5.1 u(A \cdot B) \in U(H)_{1}$. By Lemmas 5.1 and 2.1, $y\left(A_{n}, B_{n}\right)^{2}=\Delta\left(u\left(A_{n} \cdot B_{n}\right)\right)$ $\rightarrow \Delta(u(A \cdot B))$. But by the continuity of $Y(\cdot)$, (Theorem 4.2), $y\left(A_{n}, B_{n}\right)$ $\rightarrow y(A, B)$.

6. Symplectic transformations of field observables and states. 6.1. We apply the foregoing, (especially Theorem 4.1) to answer some mathematical questions in the theory of free boson fields as formulated in [11] and [12]. We begin by giving some relevant definitions and results. It is assumed that $\operatorname{dim} H$ is infinite.

Let $R(\cdot)$ be a quantization of the single particle structure $\Sigma(H)$. For any subspace $H^{\prime}$ of $H$, let $\mathfrak{A}_{H^{\prime}}$, be the weakly closed ring generated by $\left\{\exp (i R(z)): z \in H^{\prime}\right\}$. Let $\mathfrak{A}_{0}=\bigcup \mathfrak{A}_{H^{\prime}}$, the union being taken over all $H^{\prime}$ with $\operatorname{dim} H^{\prime}$ finite. The algebra of field observables $\mathfrak{A}$ is the uniform closure of $\mathfrak{A}_{0}$. The elements of $\mathfrak{A}_{0}$ are called tame field observables. If $X \in \mathfrak{A}_{H^{\prime}}$ with $\operatorname{dim} H^{\prime}$ finite then $X$ is said to be based on $H^{\prime}$. When $\operatorname{dim} H$ is infinite there are continuumly many unitarily inequivalent irreducible quantizations. However as an abstract $C^{*}$-algebra, $\mathfrak{A}$ is independent of the particular quantization used in its definition [11, Theorem 1].

A (mathematical) state $E$ of the field is a positive linear functional on $\mathscr{A}$ such that $E(I)=1$. $E$ is pure if it is not a nontrivial convex combination of distinct states. $E$ is regular if for $A, B \in \mathfrak{A}, E\left(A^{*} \cdot \exp (i R(z)) \cdot B\right)$ is continuous on each finite dimensional subspace of $H$. Our concern is with pure regular 
states. As is well known any pure state determines an irreducible representation of $\mathfrak{A}$. If $E$ is also regular it determines an irreducible quantization. Conversely let $R(\cdot)$ be an irreducible quantization on $\mathfrak{S}$. If $x \in \mathfrak{E}$ the state $E: E(X)=(X x, x)$ is pure regular and $R(\cdot)$ is the quantization it determines. This suggests, [cf. [12])

Definition 6.1. Two pure regular states $E, F$ are relatively normalizable; $E \sim F$; if there is an irreducible quantization $R(\cdot)$ on $\mathfrak{W}$ and $x, y \in \mathfrak{W}$ such that $E(X)=(X x, x)$ and $F(X)=(X y, y)$ for all $X \in \mathfrak{A}$.

Clearly $\sim \sim$ is an equivalence relation and the equivalence classes are in 1-1 correspondence with the classes of irreducible quantizations under unitary equivalence. Now let $E, F$ be determined by vectors $x, y \in \mathfrak{S}$, where $R(\cdot)$ is an irreducible quantization on $\mathfrak{S}$, then by a theorem of Kadison [6], there is an $A \in \mathfrak{A}$, such that $A x=y$. Then $F(X)=(X y, y)=(X \cdot A x, A x)=E\left(A^{*} \cdot X \cdot A\right)$. Consequently $E$ and $F$ are relatively normalizable if and only if $F(X)$ $=E\left(A^{*} \cdot X \cdot A\right)$ for all $X$ and some fixed $A \in \mathfrak{A}$. It seems plausible that only states normalizable with regard to the physical vacuum state can correspond to actual "physically realizable" states of the field.

If $T \in S p(K)$, then $\theta(T)$ is the unique *automorphism of such that $\theta(T) \exp (i R(z))=\exp (i R(T z))$ for all $z \in H$. Any ${ }^{*}$-automorphism $\theta$ of $\mathfrak{A}$ determines an automorphism $\theta^{*}$ of the states defined by $\theta^{*} E(X)=E\left(\theta^{-1} X\right)$. Both $\theta(\cdot)$ and $\theta^{*}(\cdot)$ are multiplicative. Clearly for $T \in S p(K), \theta^{*}(T)$ leaves the set of pure regular states invariant.

THEOREM 6.1. (a) If E and $F$ are pure regular states and $E \sim F$ and $T \in S p(K)$ then $\theta^{*}(T) E \sim \theta^{*}(T) F$. (b) Let $E$ be a pure regular state and $T \in S p(K)$. Let $R(\cdot)$ on $\mathfrak{S}$ and $R^{\prime}(\cdot)$ on $\mathfrak{S}^{\prime}$ be representatives of the equivalence class of quantizations determined by $E$ and $\theta^{*}(T) E$ respectively. Then there exists a unitary map $Y$ from $\mathfrak{S}$ onto $\mathfrak{S}^{\prime}$ such that $Y \cdot R(z)=R^{\prime}(T z) \cdot Y$ for all $z \in H$. (c) With $E, T$, $R(\cdot)$ and $\mathfrak{S}$ as in (b), $E \sim \theta^{*}(T) E$ if and only if there exists a unitary operator $Y$ on $\mathfrak{E}$ such that $Y \cdot R(z) \cdot Y^{-1}=R(T z)$ all $z \in H$.

Proof. (a) Since $E \sim F$, we have $F(X)=E\left(A^{*} \cdot X \cdot A\right)$ for all $X$ and some $A$ in $\mathfrak{A}$. But then $\theta^{*}(T) F(X)=\theta^{*}(T) E\left(\theta(T) A^{*} \cdot X \cdot \theta(T) A\right)$. Therefore $\theta^{*}(T) E$ $\sim \theta^{*}(T) F$. (b) We take $\mathfrak{A}$ to be realised on $\mathfrak{S}$. If $A \in \mathfrak{A}, A^{\prime}$ denotes the same observable realised as an operator on $\mathfrak{S}^{\prime}$. Let $x \in \mathfrak{S}$ and $y \in \mathfrak{S}^{\prime}$ be unit vectors determining the states $E$ and $\theta^{*}(T) E$ respectively. Let $Y(A x)=\theta(T) A^{\prime} y$. Then by the result of Kadison cited above, $Y$ is everywhere defined and maps onto $\mathfrak{S}^{\prime}$. To see that $Y$ is well defined suppose $A x=B x(B \in \mathfrak{Y})$. Let $C=A-B\|Y(C x)\|^{2}=\left(\theta(T) C^{\prime} y, \theta(T) C^{\prime} y\right)=F\left(\theta(T)\left(C^{*} \cdot C\right)\right)=E\left(C^{*} \cdot C\right)=\|C x\|^{2}$. Hence $Y(A x)-Y(B x)=0$. Replacing $C$ by $A$ above we see that $Y$ is unitary. Finally $Y(\exp (i R(z)) \cdot A x)=\theta(T)\left(\exp \left(i R^{\prime}(z)\right) \cdot A^{\prime}\right) y=\exp \left(i R^{\prime}(T z)\right) \cdot \theta(T) A^{\prime} y$. (c) Part (c) follows readily from (b) and proof is omitted.

6.2. Now let $R(\cdot)$ be the Fock-Cook quantization on $L_{2}(M, n)$. The zerointeraction vacuum state $E_{0}$ is defined by $E_{0}(X)=\left(X I_{0}, I_{0}\right)$ for all $X \in \mathfrak{A}$. It 
is the unique state invariant under the action of $U(H)$ and for which the free field energy is non-negative [13]. We have immediately by Theorem 4.1 and Lemma 6.1(c)

Corollary 6.1.1. $E_{0} \sim \theta^{*}(T) E_{0}$ if and only if $T \in r S p(K)$.

This answers a question raised in [12].

The class of pure regular states most easily treated mathematically, is $S r_{0}$, the states symplectically related to $E_{0}$. Specifically $S r_{0}=\left\{E: E \sim \theta^{*}(T) E_{0}\right.$, for some $T \in S p(K)\}$. It follows from a result of Feldman [4], that there are pure regular states not in $S r_{0}$. Now if $E \sim \theta^{*}(T) E_{0}$ and $F \sim \theta^{*}\left(T^{\prime}\right) E_{0}$, then $E \sim F$ if and only if $\theta^{*}\left(T^{-1} \cdot T^{\prime}\right) E_{0} \sim E_{0}$, or equivalently (by Corollary 6.1.1) if and only if $T^{-1} \cdot T^{\prime} \in r S p(K)$. It follows that the equivalence classes in $S r_{0}$ are in 1-1 correspondence with the elements of the space $\subseteq$ of left cosets of $r S p(K)$ in $S p(K)$. For $\bar{T} \in \mathbb{S}$ let $E(\bar{T})$ be the corresponding class of pure regular states. Let $R_{\bar{T}}(\cdot)$ on $\mathfrak{S}_{\bar{T}}$ be a representative of the class of unitarily equivalent irreducible quantizations determined by $E(\bar{T})$. Extending Theorem 4.1 we have immediately:

CoRollary 6.1.2. Let $T^{\prime} \in S p(K)$. There is a unitary operator $Y$ on $\mathfrak{S}_{\bar{T}}$ such that $R_{\bar{T}}\left(T^{\prime} z\right)=Y \cdot R_{\bar{T}}(z) \cdot Y^{-1}$ for all $z \in K$, if and only if $T^{\prime} \in \bar{T} \cdot r S p(K)$ $\cdot \bar{T}^{-1}$.

REMARK 6.1. Given $T \in S p(K)$ it is an open question whether there exists irreducible quantization $R(\cdot)$ and a unitary operator $Y$ such that $R(T z)$ $=Y \cdot R(z) \cdot Y^{-1}$ for all $z \in K$. However if irreducibility is not required such a $Y$ may be constructed, using Lemma 6.1 (b), with $R(\cdot)=\oplus \sum R_{\bar{T}}(\cdot)$ on $\mathfrak{Q}=\oplus \sum \mathfrak{S}_{\bar{T}}$, where the summations are over $\mathfrak{S}$. It is not clear however that a projective unitary representation of $S p(K)$ can be obtained. It is moreover quite hopeless to attempt to recover "observables" from 1-parameter subgroups in $S p(K)$ in the manner discussed in Remark 4.1. For let $T(\lambda)$ $=\lambda^{-1} I \oplus \lambda I$ on $K=M \oplus M, \lambda \neq 0$.

Corollary 6.13. For any $\lambda \neq 0,1$, and any $E \in S r_{0}, \theta^{*}(T(\lambda)) E$ and $E$ are not relatively normalizable.

Proof. Suppose $E \sim \theta^{*}(T) E_{0}$. By Corollary 6.1.2 $\theta^{*}(T(\lambda)) E \sim E$ if and only if $T^{-1} \cdot T(\lambda) \cdot T \in r S p(K)$. Suppose $T^{-1} \cdot T(\lambda) \cdot T \in r S p(K)$. Then $T^{*} \cdot T(\lambda)$ $\cdot T^{*-1} \cdot T^{-1} \cdot T(\lambda) \cdot T \in S p(K)_{2}^{+}$. Let $A=\left(T \cdot T^{*}\right)^{-1}$. Then since $S p(K)_{2}$ is a normal subgroup of $S p(K)$, we have $A^{-1} \cdot T(\lambda) \cdot A \cdot T(\lambda) \in S p(K)_{2}$. Therefore $C=T(\lambda)^{-1} \cdot A-A \cdot T(\lambda)$ is Hilbert-Schmidt. Let $P$ be the projection on $K=M \oplus M$ such that $P(x \oplus y)=0 \oplus y$. Then $C \cdot P=\left(\lambda^{-1}-\lambda\right) P \cdot A \cdot P$ and, if $\lambda \neq 1, P \cdot A \cdot P$ are Hilbert-Schmidt. But $A$ is selfadjoint and strictly positive since $A^{-1}$ is bounded. Further, the range of $P$ has infinite dimension. Hence $P \cdot A \cdot P$ cannot be Hilbert-Schmidt. Contradiction. 
6.3. In view of the foregoing it becomes natural to enquire for which $T \in S p(K)$ the field automorphisms $\theta(T)$ are inducible by a unitary operator in every irreducible quantization. The following two theorems give partial answers.

TheOREM 6.2. For $T \in S p(K), \theta(T)$ is inducible by a unitary operator in all quantizations arising from states symplectically related to the zero interaction vacuum state $E_{0}$, if and only if $T= \pm I+X$ with $X$ Hilbert-Schmidt.

Proof. By Corollary 6.1.2, $T$ has the desired property if and only if $T \in G=\left\{T \in S p(K): T^{\prime-1} \cdot T \cdot T^{\prime} \in r S p(K)\right.$ for all $\left.T^{\prime} \in S p(K)\right\}$. Let $G^{\prime}=\{T \in S p(K): T= \pm I+X$ with $X$ Hilbert-Schmidt $\}$. Then $G^{\prime}$ is a normal subgroup of $S p(K)$ and $G^{\prime} \subset r S p(K)$. Therefore $G^{\prime} \subset G$. If $T \in G \subset r S p(K)$, then $|T| \in S p(K)_{2} \subset G^{\prime} \subset G$. Therefore $u(T) \in G$ also. Consequently it is sufficient to show that if $T$ is unitary and $T \in G$ then $T \in G^{\prime}$. We show first that $T^{-1}-T$ is Hilbert-Schmidt. Now $T=\exp (i A)$ with $A$ bounded and selfadjoint. There is no loss in supposing that $A$ leaves $M$ invariant. Then on $K=M \oplus M, T$ corresponds to the matrix

$$
\left(\begin{array}{rr}
\cos A & -\sin A \\
\sin A & \cos A
\end{array}\right) \text {. }
$$

Now let $T(\lambda)$ be as defined in Corollary 6.1.3. Then $T(\lambda)^{-1} \cdot T \cdot T(\lambda) \in r S p(K)$. Therefore $T(\lambda) \cdot T^{*} \cdot T\left(\lambda^{-2}\right) \cdot T \cdot T(\lambda) \in S p(K)_{2}^{+}$. Therefore $C=T\left(\lambda^{2}\right) \cdot T^{*} \cdot T\left(\lambda^{-2}\right)$ - $T \in S p(K)_{2}$. A computation gives the matrix corresponding to $C-I$ as

$$
\left(\begin{array}{cc}
\lambda^{-4}-1 & 0 \\
0 & \lambda^{4}-1
\end{array}\right)\left(\begin{array}{cc}
\sin A & 0 \\
0 & \sin A
\end{array}\right)\left(\begin{array}{rr}
\sin A & \cos A \\
-\cos A & \sin A
\end{array}\right) \text {. }
$$

But $C-I$ is Hilbert-Schmidt. It follows that $\sin A$ and hence $T^{-1}-T$ are Hilbert-Schmidt. Hence $T^{2}=I+X$ with $X$ Hilbert-Schmidt. It follows that $T$ has one of the three following forms: $I+X_{1}$ on $H,-I+X_{1}$ on $H$, or $\left(I+X_{1}\right) \oplus\left(-I+X_{2}\right)$ on $H=H_{1} \oplus H_{2}$ where $X_{1}$ and $X_{2}$ are Hilbert-Schmidt and $\operatorname{dim} H_{1}$ and $\operatorname{dim} H_{2}$ are infinite. Suppose $T$ has the latter form and $T \in G$. By further decomposing $H_{1}$ and $H_{2}$ it may be seen that there is no loss in supposing further that $\operatorname{dim} H_{1}=\operatorname{dim} H_{2}$ and that neither 1 or -1 is in the spectrum of $X_{1}$ or $X_{2}$. Then $\left(I+X_{1}\right) \oplus\left(I-X_{2}\right) \in G^{\prime}$ and $T^{\prime}=I \oplus-I \in G$. For $z=x \oplus y$ in $H=H_{1} \oplus H_{2}$, let $S(t) z=(\cos t \cdot x-\sin t \cdot y) \oplus(\sin t \cdot x+\cos t \cdot y)$. Then $S(t) \in U(H)$ and $S(2 t)=T^{\prime} S(t)^{-1} \cdot T^{\prime} \cdot S(t) \in G$. But $\left(S(2 t)^{-1}-S(2 t)\right) z$ $=2 \sin 2 t \cdot y \oplus-2 \sin 2 t \cdot x$ which is not Hilbert-Schmidt unless $\sin 2 t=0$. Contradiction. This proves the theorem.

A *-automorphism $\theta$ of $\mathfrak{A}$ is inner if there is a $Y \in \mathfrak{A}$ such that $\theta X=Y \cdot X \cdot Y^{-1}$ for all $X \in \mathfrak{A}$. Then $\theta X=\left(\theta X^{*}\right)^{*}=Y^{*-1} \cdot X \cdot Y^{*}$. It follows that $Y^{*} \cdot Y$ is in the center of $\mathfrak{A}$. It may be assumed without loss that $Y^{*} \cdot Y=I$. Consequently 
an inner automorphism is inducible by a unitary operator in every quantization.

TheOREM 6.3. For $T \in S p(K), \theta(T)$ is inner if and only if $T \in S p(K)_{0}$ (i.e. $T$ is tame).

Proof. Let $R(\cdot)$ be the Fock-Cook quantization on $L_{2}(M, n)$. If $T \in S p(K)_{0}$, it follows from Definition 4.1 that $Y(T) \in \mathfrak{A}_{0}$. If $\theta(T)$ is inner, $T \in r S_{p}(K)$ and we may suppose that $Y=Y(T)$. Now $\mathfrak{A}_{0}$ is stable under polar decomposition. Hence given $\epsilon>0$, there exists a unitary operator $U \in \mathfrak{A}_{0}$ and based on a subspace $H^{\prime}$ of $H$ with $\operatorname{dim} H^{\prime}$ finite, such that $\|Y-U\|<\epsilon$ and $\left\|Y^{-1}-U^{-1}\right\|<\epsilon$. Then for all $z \in K,\left\|U \cdot \exp (i R(z)) \cdot U^{-1}-\exp (i R(T z))\right\|=\| U \cdot \exp (i R(z)) \cdot U^{-1}$ $-Y \cdot \exp (i R(z)) \cdot Y^{-1} \|$ which may be seen to be less than $2 \epsilon$. Now let $H^{\prime \prime}=H^{\prime \perp}$ and $K^{\prime}$ and $K^{\prime \prime}$ be subspaces in $K$ and $M^{\prime}$ and $M^{\prime \prime}$ be subspaces in $M$ corresponding to $H^{\prime}$ and $H^{\prime \prime}$ respectively. Since on $L_{2}\left(M^{\prime}, n\right) \otimes L_{2}\left(M^{\prime \prime}, n\right) U$ decomposes as $U^{\prime} \otimes I$, it follows that $U$ commutes with $\exp (i R(z))$ for all $z \in K^{\prime \prime}$. Hence for $z \in K^{\prime \prime}, \| \exp (i R(i j)-\exp (i R(T z)) \|<2 \epsilon$.

Suppose $\epsilon<1 / 2$. Then $T=I$ on $K^{\prime \prime}$. Otherwise there is a unit vector $z_{2}=z_{1}-T z_{1}$ with $z_{1} \in K^{\prime \prime}$. Then using the commutation relations (1.1), for any integer $n$, we have $\left|\left(\exp \left(i R\left(n z_{1}\right)\right) I_{0}, \exp \left(i R\left(n T z_{1}\right)\right) I_{0}\right)\right|=\left|\left(\exp \left(i R\left(n z_{2}\right)\right) I_{0}, I_{0}\right)\right|$. But since $\theta^{*}(V) E_{0}=E_{0}$ for all $V \in U(H)$, this equals $\left|\left(\exp \left(i R\left(n V z_{2}\right)\right) I_{0}, I_{0}\right)\right|$. Now let $V$ be such that on $K=M \oplus M V z_{2}=0 \oplus-y$. Then with the notation of $\$ 4.1, R\left(n V z_{2}\right)=n Q(y)$. By [7, Corollary 3.4], $Q(y)$ is the operation of multiplication by $(\cdot, y) /(2 c)^{1 / 2}$ on $L_{2}(M, n)$. Consequently $\left(\exp (\operatorname{in} Q(Y)) I_{0}, I_{0}\right)$ may be evaluated as $\left(1 /(2 \pi c)^{1 / 2}\right) \int \exp \left(\left(i n y /(2 c)^{1 / 2}\right)-y^{2} / 2 c\right) d y,=\exp \left(-n^{2} / 4\right)$ the integral being taken over the reals with regard to Lebesgue measure. We have for $z \in K^{\prime \prime}$,

$$
\begin{aligned}
\sup & \|\exp (i R(z))-\exp (i R(T z))\|^{2} \\
& \geqq\left\|\left(\exp \left(i R\left(n z_{1}\right)\right)-\exp \left(i R\left(n T z_{1}\right)\right)\right) I_{0}\right\|^{2} \\
& =2-2 \operatorname{Real}\left(\left(\exp \left(i R\left(n z_{1}\right)\right) I_{0}, \exp \left(i R\left(n T z_{1}\right)\right) I_{0}\right)\right) \\
& \geqq 2-2 \exp \left(-n^{2} / 4\right), \quad \text { for all } n .
\end{aligned}
$$

Hence for $z \in K^{\prime}, \sup \|\exp (i R(z))-\exp (i R(T z))\|^{2} \geqq 2$. Contradiction. This shows that $T=I$ on $K^{\prime \prime}$.

Let $K^{\prime \prime \prime}=K^{\prime} \cup T\left(K^{\prime}\right)$. Then $\operatorname{dim} K^{\prime \prime \prime}$ is finite. It may be seen that $K^{\prime \prime \prime}$ is invariant under $T$. Also since $\left(K^{\prime \prime \prime}\right)^{\perp} \subset K^{\prime \prime}, T=I$ on $\left(K^{\prime \prime \prime}\right)^{\perp}$. Therefore $T$ is based on $K^{\prime \prime \prime}$ and $T \in S p(K)_{0}$. This proves the theorem.

\section{REFERENCES}

1. V. Bargmann, On unitary ray representations of continuous groups, Ann. of Math. 59 (1954), 1-46.

2. R. Brauer and H. Weyl, Spinors in $n$ dimensions, Amer. J. Math. 57 (1935), 425-449.

3. J. M. Cook, The mathematics of second quantization, Trans. Amer. Math. Soc. 74 (1953), 222-245. 
4. J. Feldman, Examples of non-gaussian quasi-invariant distributions in Hilbert space, Trans. Amer. Math. Soc. 99 (1961), 342-349.

5. Harish-Chandra, Representations of a semisimple Lie group on a Banach space. I, Trans. Amer. Math. Soc. 75 (1953), 185-243.

6. R. V. Kadison, Irreducible operator algebras, Proc. Nat. Acad. Sci. U.S.A. 43 (1957), 273-276.

7. I. E. Segal, Tensor algebras over Hilbert spaces. I, Trans. Amer. Math. Soc. 81 (1956), 106-134.

8. - Ergodic subgroups of the orthogonal group on a real Hilbert space, Ann. of Math. 66 (1957), 297-303.

9. - The structure of a class of representations of a unitary group on a Hilbert space, Proc. Amer. Math. Soc. 8 (1957), 197-203.

10. - Distributions in Hilbert space and canonical systems of operators, Trans. Amer. Math. Soc. 88 (1958), 12-41.

11. - Foundations of the theory of dynamical systems of infinitely many degrees of freedom. I, Mat. Fys. Medd. Danske Vid. Selsk. 31, no. 12 (1959).

12. - Foundations of the theory of dynamical systems of infinitely many degrees of freedom. II, Canad. J. Math. 13 (1961), 1-19.

13. - The characterisation of the physical vacuum, to appear.

14. B. Sz.Nagy, Spectraldarstellungen linearer Transformationen des hilbertschen Räume, Springer-Verlag, Berlin, 1942.

15. L. van Hove, Sur certaines représentations unitaires d'un groupe infini de transformations, Mémoires de Acad. Roy. de Belg. No. 1618 (1951).

16. J. von Neumann, Die Eindeutigkeit der Schrödingerschen Operatoren, Ann. Mat. Pura Appl. 104 (1931), 570-578.

17. H. Weyl, Gruppentheorie und Quantenmechanik, 2d ed., Leipzig, 1931.

18. N. Wiener, The Fourier integral, Dover, New York, 1951.

University of Chicago,

Chicago, Illinois

UNIVERSITY OF TORONTO,

TORONTO, ONTARIO 